hampered research efforts, resulting in remarkably limited therapeutic evidence. The EuroMyositis Registry was created to pool resources and expertise across the international IIM research community.

Objectives: To describe the phenotypic characteristics of different IIM subtypes. Associations with malignancy, interstitial lung disease (ILD), cardiac involvement and dysphagia were assessed.

Methods: Pooled data from the EuroMyositis Registry (from Belgium, China, Czech Republic, Hungary, Italy, Mexico, Norway, Sweden, Switzerland, UK, Vietnam) were obtained. Associations were assessed using logistic regression and multinomial logistic regression with polymyositis (PM) as the reference group. Results: Data regarding 3,196 patients were analysed. The UK was the largest contributor $(n=1,307)$. The most common diagnoses were dermatomyositis (34\%), PM (32\%) and connective tissue disease (CTD)-overlap myositis (15\%). In those with anti-synthetase syndrome $(7 \%), 85 \%$ had muscle weakness, $86 \%$ ILD and $58 \%$ arthritis. Overall, $43 \%$ had a myositis specific antibody, most commonly anti-Jo1 autoantibodies (20\%). Glucocorticoid usage was noted in $98 \%$. Most commonly used disease modifying agents were methotrexate $(71 \%)$ and azathioprine (50\%)

Malignancy occurred in $9 \%$ and was associated with a diagnosis of dermatomyositis (Relative Risk Ratio [RRR] 1.68, 95\% Cl 1.09-2.56, $\mathrm{p}=0.018$ ). Cardiac involvement occurred in $9 \%$, most commonly in those with CTD-overlap myositis (13\%), and was associated with a higher Health Assessment Questionnaire disability index (1 versus 0.75 , OR $1.40,95 \% \mathrm{Cl} 1.03-1.91, \mathrm{p}=0.031$ ). Dysphagia occurred in $39 \%$ and was associated with a diagnosis of CTD-overlap myositis (RRR 2.25, 95\% Cl 1.61-3.15, p<0.001).

Conclusions: This large international cohort demonstrates the heterogeneity of IIM and the burden of associated malignancy, ILD, cardiac and gastrointestinal involvement. The EuroMyositis Registry facilitates international collaborative research outputs, underlining the benefits of harmonised data collection methodology between centres.

Acknowledgements: This work was supported by researchers at the National Institute for Health Research (NIHR) Biomedical Research Unit. The views expressed are those of the authors and not necessarily those of the UK National Health Service, the NIHR or the UK Department of Health

Disclosure of Interest: J. Lilleker: None declared, J. Vencovsky: None declared, G. Wang: None declared, L. Wedderburn Grant/research support from: The UK JDM Cohort and Biomarker study is supported by grants from the NIHR and Myositis UK, L. Diederichsen: None declared, J. Schmidt: None declared, P. Jordan: None declared, O. Benveniste: None declared, M. G. Danieli: None declared, K. Dankó: None declared, N. T. P. Thuy: None declared, M. VazquezDel Mercado: None declared, $\varnothing$. Molberg: None declared, B. De Paepe: None declared, J. De Bleecker: None declared, B. Maurer Grant/research support from: AbbVie, Protagen, EMDO, Novartis, Roche, Actelion, N. Pipitone Speakers bureau: GRAPPA Workshop, Alfa-Wassermann, N. McHugh: None declared, Z. Betteridge: None declared, P. New: None declared, R. Cooper: None declared, W. Ollier: None declared, J. Lamb Grant/research support from: Medlmmune, N. S. Krogh: None declared, I. Lundberg Grant/research support from: Astra-Zeneca, Bristol-Myers Squibb, Consultant for: Bristol-Myers Squibb, Idera, H. Chinoy Grant/research support from: Medlmmune, Novartis

DOI: 10.1136/annrheumdis-2017-eular.2742

\section{SAT0375 METABOLOMICS OF SERA REVEALS POTENTIAL BIOMARKERS OF SKIN FIBROSIS IN SYSTEMIC SCLEROSIS THAT CORRELATE WITH PRO-FIBROTIC GENE EXPRESSION IN SKIN BIOPSIES}

S. Ahmed ${ }^{1}$, M.K. Rai ${ }^{1}$, D. Dubey ${ }^{2}$, A. Rawat ${ }^{2}$, D.P. Misra ${ }^{1}$, D. Kumar ${ }^{2}$, V. Agarwal ${ }^{1} .{ }^{1}$ Clinical Immunology, Sanjay Gandhi Post Graduate Institute of Medical Sciences; ${ }^{2}$ Centre for BioMedical Research, Lucknow, India

Background: There is an unmet need for biomarkers in Systemic Sclerosis (SSc). Despite its shortcomings, the modified Rodnan skin score (mRSS) has remained the standard disease assessment tool for SSc. Expression of certain genes, cartilage oligomeric matrix protein (COMP), thrombospondin-1 (THS1), interferon-induced 44 (IFI44) and sialoadhesin (SIG1), and, more recently, Tenascin-C (TNSC), have been shown to correlate with skin fibrosis. However, assessment of the expression of these genes requires a skin biopsy. Hence, we used an open-ended approach to identify a serum-based biomarker of SSc.

Objectives: To identify small molecules in serum that correlate with mRSS and profibrotic genes that are upregulated in skin of SSc patients

Methods: We obtained serum and skin biopsies from 25 consenting adult patients with SSc and serum from 25 age- and sex-similar controls. mRNA levels of five genes: COMP, THS1, IFI44, SIG1, and TNSC were estimated as fold-change relative to Glyceraldehyde 3-phosphate dehydrogenase (GAPDH), a housekeeping gene. $\mathrm{H}^{1} \mathrm{NMR}$ (Nuclear Magnetic Resonance) based metabolomics studies were performed on the sera using standard protocols. Principal component analysis (PCoA) and Partial Least Squares Discriminant Analysis (PLSDA) were used to delineate metabolites that were different between patients and healthy controls. Then spearman correlations $(\rho)$ of these metabolites with mRSS and the fold-expression of the five pro-fibrotic genes were estimated.

Results: $\mathrm{H}^{1} \mathrm{NMR}$ based metabolomics identified 126 peaks that were different between patients and controls. Out of these, the levels of glycine had the best correlation with pro-fibrotic gene expression $(\rho=0.5, p<0.05$ for IFI44; $\rho=0.44$,
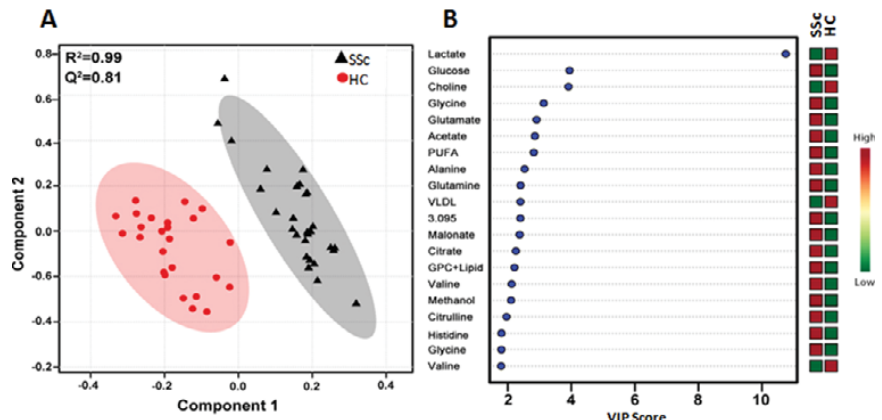

Figure 1: $\mathrm{H}^{1}$-NMR Metabolomics data (A) PCOA plot showing metabolomics can clearly distinguish between patients of systemic sclerosis and healthy controls (B) Plot showing 20 representative metabolites that were significantly different between patients and controls; SSc: Systemic Sclerosis, HC: Healthy Controls, PUFA: Poly Unsaturated Fatty Acids; VLDL: Very Low Density Lipoproteins, GPC: Glycerophosphocholine, VIP: Variable Importance in Projection Score

$\mathrm{p}<0.05$ for TNSC). Choline inversely correlated with SIG1 $(\rho=-0.41 ; \mathrm{p}=0.05)$ while Glycerophosphocholine correlated with $\operatorname{mRSS}(\rho=0.50, p<0.05)$.

Conclusions: $\mathrm{H}^{1} \mathrm{NMR}$ based metabolomics identified glycine, choline and their metabolites as potential biomarkers for skin fibrosis in SSc. These findings require validation in a larger cohort

References:

[1] Farina G, Lafyatis D, Lemaire R, Lafyatis R. A four-gene biomarker predicts skin disease in patients with diffuse cutaneous systemic sclerosis. Arthritis Rheum. 2010 Feb;62(2):580-8.

[2] Bhattacharyya S, Wang W, Morales-Nebreda L, Feng G, Wu M, Zhou X, Lafyatis R, Lee J, Hinchcliff M, Feghali-Bostwick C, Lakota K, Budinger GR, Raparia K, Tamaki Z, Varga J.Tenascin-C drives persistence of organ fibrosis. Nat Commun. 2016 Jun 3:7:11703.

Disclosure of Interest: None declared

DOI: 10.1136/annrheumdis-2017-eular.3715

\section{SAT0376 ANTI-NT5C1A AUTOANTIBODIES ARE FREQUENT IN JUVENILE MYOSITIS AND ASSOCIATED WITH INCREASED ILLNESS SEVERITY}

R. Yeker ${ }^{1}$, I. Pinal-Fernandez ${ }^{1}$, T. Kishi ${ }^{2}$, I.N. Targoff ${ }^{3}$, F.W. Miller ${ }^{2}$, L.G. Rider $^{2}$ A. Mammen ${ }^{1} .{ }^{1}$ Muscle Disease Unit, National Institute of Arthritis and Musculoskeletal and Skin Diseases (NIAMS)/National Institutes of Health (NIH); ${ }^{2}$ Environmental Autoimmunity Group, National Institute of Environmental Health Sciences (NIEHS)/National Institutes of Health $(\mathrm{NIH})$, Bethesda; ${ }^{3}$ University of Oklahoma Health Sciences Center, Oklahoma City, United States

Background: Autoantibodies (Abs) against 5'-Nucleotidase, Cytosolic 1A (NT5C1A) have been portrayed as a potential diagnostic aide in distinguishing inclusion body myositis (IBM) and polymyositis (PM) in adults. However, $4-21 \%$ of dermatomyositis (DM) patients also have Abs to NT5C1A.

Objectives: The prevalence and clinical features of anti-NT5C1A Abs in juvenileonset myositis (IIM) patients is unknown, so we sought to examine this in a large cohort.

Methods: We screened 384 juvenile IIM patients meeting probable or definite Bohan and Peter criteria for anti-NT5C1A Abs by immunoblotting for the full length NT5C1A protein in transfected and non-transfected lysates. Clinical characteristics and HLA typing were compared between juvenile IIM patients who were anti-NT5C1A positive $(A b+)$ and those who were anti-NT5C1A negative $(\mathrm{Ab}-)$

Results: In this cohort, $29 \%$ (103) of juvenile DM, $15 \%$ (3) of juvenile PM, and $37 \%(15)$ of juvenile overlap myositis patients tested positive for anti-NT5C1A Abs. Compared with anti-NT5C1A Ab-patients, anti-NT5C1A Ab+ juvenile IIM patients showed a similar distribution of race, gender, and association with myositis-specific Abs. However, NT5C1A Ab was associated with anti-p155/140 Abs $(p=0.05)$. The only observed clinical difference was an increased frequency of $\mathrm{V}$ - or shawl-sign rashes ever present $(44 \%$ vs. $26 \%, p=0.002)$. Disease severity was increased in anti-NT5C1A Ab+ patients, based on more frequent hospitalizations $(p=0.02)$, more medications used $(p<0.001)$, and more treatment trials per year $(p<0.001)$. Additionally, pulse steroids $(p<0.001)$ and intravenous immunoglobulin therapy $(p=0.008)$ were prescribed more frequently in anti-NT5C1A Ab+than Ab- patients. The HLA alleles DRB1*07 (20\% vs. $9 \%, p<0.05)$ and DQA $1^{*} 0201(21 \%$ vs. $7 \%$, $p<0.01$ ) were present more frequently in anti-NT5C1A Ab- compared to Abpatients.

Conclusions: Anti-NT5C1A Abs are commonly present in juvenile DM and juvenile overlap myositis patients, and are present more frequently in patients with anti-p155/140 Abs, but are also seen in association with other myositis specific Abs. Consistent with data in adult IIM patients, anti-NT5C1A Abs have few distinguishing clinical features in juvenile myositis, but are associated with increased illness severity marked by increased hospitalizations and receipt of additional therapy.

Disclosure of Interest: R. Yeker: None declared, I. Pinal-Fernandez Grant/ research support from: The Myositis Association, T. Kishi Grant/research support 
from: The Myositis Association, I. Targoff Consultant for: Oklahoma Medical Research Foundation Clinical Immunology Laboratory regarding myositis autoantibody testing, F. Miller: None declared, L. Rider: None declared, A. Mammen: None declared

DOI: 10.1136/annrheumdis-2017-eular.6246

\section{SAT0377 RELIABILITY OF A NEW AUTOMATED SYSTEM FOR ABSOLUTE CAPILLARY NUMBER COUNTING (AUTOCAPI) ON SYSTEMIC SCLEROSIS NAILFOLD VIDEOCAPILLAROSCOPIC IMAGES}

M. Cutolo ${ }^{1}$, K. Melsens ${ }^{2}$, A.C. Trombetta ${ }^{1}$, C. Pizzorni ${ }^{1}$, E. Deschepper ${ }^{3}$, A. Sulli ${ }^{1}$, V. Smith ${ }^{4} .{ }^{1}$ Research Laboratory and Academic Division of Clinical Rheumatology, Department of Internal Medicine, University of Genoa, Genoa, Italy; ${ }^{2}$ Department of Internal Medicine, Faculty of Medicine and Health Sciences: ${ }^{3}$ Department of Public Health, Biostatistics Unit; ${ }^{4}$ Department of Internal Medicine, Faculty of Medicine and Health Sciences; Department of Rheumatology, Ghent University Hospital, Ghent University, Ghent, Belgium

Background: Nailfold capillary density is a useful measure in systemic sclerosis (SSc) classification and evaluation. Its manual detection may be time-consuming, hampering its use in largescale investigations. We evaluated a new automated system to assess the absolute nailfold capillary number.

Objectives: To attest the instrumental reliability of automatic counting in SSc patients using nailfold video capillaroscopy (NVC) images.

Methods: 75 NVC random images, from SSc patients, were blindly analyzed by four raters (2 less and 2 more experienced; raters: 1,2,3,4) from two European centers. Each rater was asked to define the region of interest (ROI) on the NVC images and to manually count the number of capillaries, according to the following instructions: upper bound placed on top of the longest capillary head and lower bound placed on half of the length of that longest capillary (figure 1); if the common branch of an abnormal shape (neoangiogenesis) is in ROI it is counted as being one; if the common branch is out of $\mathrm{ROI}$ it is counted as separate capillaries; if the capillary is on the edge of the vertical line of ROI, it is only counted when the head of the capillary is half in ROI; if the capillary head is on the edge of lower bound, it is counted as soon as the "head" part is in the ROI; all "heads" in the ROI are counted (not only distal row). The dedicated automated system (AUTOCAPI-ds medica, IT) also counted the number of capillaries in the same ROI (figure 1). Reliability between the manual and automatic counting was investigated per rater through intraclass correlation coefficient (ICC) and reported with $95 \%$ confidence interval $(\mathrm{Cl})$. External validation was obtained by multi-rating of the same set of images. Average difference between automated and manual counting per rater was calculated.

Results: The ICC $(95 \% \mathrm{Cl})$ of manual versus automatic counting in $\mathrm{ROI}$ was 0.77 $(0.61-0.86)$ for rater $1(p<0.0001), 0.81(0.71-0.88)$ for rater $2(p<0.0001), 0.65$ $(0.50-0.76)$ for rater $3(\mathrm{p}<0.0001)$ and $0.81(0.71-0.87)$ for rater $4(\mathrm{p}<0.0001)$. The average difference was -0.69 for rater $1,0.04$ for rater $2,-0.03$ for rater 3 and 0.16 for rater 4

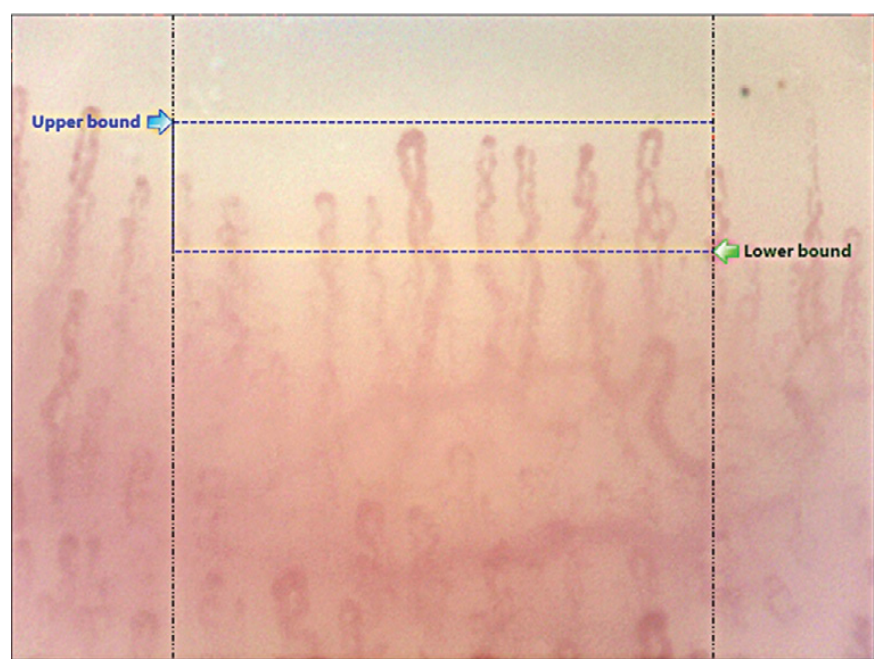

Figure 1. In the picture captured by the operator (normal subject), two vertical bars, with a distance of $1 \mathrm{~mm}$ appears automatically in the middle of the picture, together with two horizontal bars (upper bound and lower bound) to be placed manually (see text) in order to define the ROI.

Conclusions: This is a first study to attest the reliability of a new automated system to calculate the absolute number of capillaries in a ROI arising from SSc NVC images. High performance of the new automated counting system was confirmed in pathological conditions (SSc).

Disclosure of Interest: None declared

DOI: 10.1136/annrheumdis-2017-eular.3191

\section{SAT0378 BONE MARROW OEDEMA AND SYNOVITIS ON MRI OF THE HAND ARE ASSOCIATE WITH DIGITAL ULCERS, ACTIVE DISEASE AND IMPAIRED FUNCTIONAL CAPACITY IN SYSTEMIC SCLEROSIS}

B. Stamenkovic ${ }^{1,2}$, S. Stojanovic ${ }^{1,2}$, J. Nedovic ${ }^{2}$, V. Zivkovic ${ }^{1,2}$, S. Milenkovic ${ }^{2}$, J. Jovanovic ${ }^{2}$, N. Damjanov ${ }^{3}$, A. Stankovic ${ }^{1,2} .{ }^{1}$ Medical School; ${ }^{2}$ Rheumatology, Institute Niska Banja, Nis; ${ }^{3}$ Rheumatology, Institute for Rheumatology, Belgrade, Serbia

Background: Hand inflammatory involvement is a major feature in patients with systemic sclerosis (SSc), responsible for major disability. Magnetic resonance imaging (MRI) can identify and characterize subclinical inflammation and joint damage on hand with much greater precision than clinical, radiographic and ultrasonography assessment in SSc.

Objectives: To determine the association of MR bone marrow oedema, synovitis and probability for the occurrence of listed inflammatory changes with clinical features and laboratory tests in SSc patients

Methods: 112 SSc patients were tested (mean age 54y). Contrast enhanced low field MRI of the wrist and MCP2-5 joints was performed to all the pts. MRI bone marrow oedema and synovitis were assessed and scored by OMERACT RAMRIS scoring system. Age, sex, SSc type, disease duration (date of first non Raynaud symptom), Raynaud phenomenon, articular or periarticular pain, joint swelling and contractures, digital ulceration, $\mathrm{HAQ}$, acroosteolysis (by standard PA radiographs of hand and wrist) pulmonary arterial hypertension (PAP $>40 \mathrm{mmHg}$ at rest on Doppler echocardiography), pulmonary fibrosis (by CT and pulmonary function tests) and laboratory tests (antibody profile, RF, CRP, Creatine phosphokinase) and disease activity (by Valentini index) were done.

Results: By multiple logistic regression analysis taking into account all clinical and laboratory variable, we found that MRI bone marrow oedema of the hand was associated and probability for the occurance of MRI bone marrow oedema was higher for the SSc pts with digital ulcers (OR=6.081;95\%IP:1.295-28.550; $\mathrm{p}<0.05), \quad H A Q>1.5(\mathrm{OR}=6.448 ; 95 \% \mathrm{IP}: 1.714-24.257 ; \mathrm{p}<0.01)$ and active disease (OR=3.377; 95\%IP; 1.175-9.706; $p<0.05)$

MRI synovitis of the hand was associated and probability for the occurance of MRI synovitis was higher, also, for the SSc pts with digital ulcers (OR=5.128; 95\%IP: 1.085-24.243; $p<0.05$ ), HAQ>1.5 (OR=9.067; 95\%IP: 1.925-42.708; $p<0.01)$ and active disease (OR=3.565; 95\%IP: $1.181-10.764 ; \mathrm{p}<0.05)$.

Conclusions: MRI bone marrow oedema and synovitis on the hand in SSc are associate with digital ulcers, impaired functional capacity and active disease. Monitoring and treatment of clinical features and organ involvement are essential in all the pts with SSc, especially those with proven bone marrow oedema and synovitis on MRI of the hand.

Disclosure of Interest: None declared

DOI: 10.1136/annrheumdis-2017-eular.4522

\section{SAT0379 NAILFOLD CAPILLAROSCOPY FINDINGS IN PATIENTS WITH INFLAMMATORY MYOPATHY AND/OR SPECIFIC OR ASSOCIATED ANTIBODIES}

A.M. Millan Arciniegas ${ }^{1}$, M.A. Martinez ${ }^{2}$, A. Baucells ${ }^{2}$, C. Juarez ${ }^{2}$, L. Martinez ${ }^{2}$, H.S. Park ${ }^{1}$, B. Magallares ${ }^{1}$, A. Laiz ${ }^{1}$, P. Moya ${ }^{1}$, J.M. Llobet ${ }^{1}$, C. Diaz Torne ${ }^{1}$, I. Castellvi ${ }^{1} .{ }^{1}$ Rheumatology; ${ }^{2}$ Immunology, Hospital de Sant Pau, Barcelona, Spain

Background: Nailfold videocapillaroscopy (NVC) is an easy, fast and nonaggressive tool, useful in the study of autoimmune diseases. The use of NVC in Inflammatory Myopathy (IM) is not clearly established.

Objectives: 1 . To evaluate capillaroscopic findings in patients with IM and/or with presence of specific or associated antibodies with this pathology. 2. To analyze possible relationships with clinical characteristics of the patients.

Methods: Retrospective review of a cohort of patients with IM and/or with presence of specific or associated antibodies, followed in Rheumatology Unit of a University Hospital.

Patients underwent a NVC at 200x, being evaluated for the presence of: loss of capillary density, enlarged and giant capillaries, ramified capillaries, haemorrhages, thrombosis, tortuous capillaries, avascular areas, disorganization of capillary architecture and subpapilar venous plexus. The following variables were also collected: sex, age, active smoking, muscle weakness, CK elevation at diagnosis, compatible muscle EMG and biopsy, skin findings, cardiac disease, dysphagia, lung disease, Raynaud's phenomenon, cancer history and overlap syndromes.

For the comparison of qualitative and/or quantitative variables Fisher's exact Test or T-test was performed when necessary.

Results: Twenty patients with at least one NVC (45\% with 2), $65 \%$ female, with a mean age of 58 years \pm 11.6 were evaluated. The characteristics of the patients are detailed in table 1 .

$65 \%$ of patients had some capillaroscopic alteration. The findings in NVC-1 and NVC-2 were: loss of capillary density $30 \%$ and $33 \%$, tortuous capillaries $90 \%$ and $89 \%$, enlarged capillaries $65 \%$ and $66.7 \%$ (giants $30 \%$ and $33 \%$ ), ramifications $40 \%$ and $55.6 \%$, disorganization $10 \%$ and $33 \%$, haemorrhages $25 \%$ and $44 \%$, thrombosis $20 \%$ and $0 \%$, avascular areas $25 \%$ and $22 \%$, visible venous plexus $40 \%$ and $55 \%$.

The presence of dysphagia was associated with the presence of loss of capillary 